\title{
Dynamical correlations in multiorbital Hubbard models: fluctuation exchange approximations
}

\author{
V Drchal ${ }^{1}$, V Janiš ${ }^{1}, \mathbf{J}$ Kudrnovský ${ }^{1}$, V S Oudovenko ${ }^{2,3}$, X Dai $^{3}$, K Haule $^{3}$ \\ and $\mathbf{G}$ Kotliar $^{3}$ \\ ${ }^{1}$ Institute of Physics, AS CR, Na Slovance 2, CZ-182 21 Praha 8, Czech Republic \\ 2 Bogoliubov Laboratory for Theoretical Physics, Joint Institute for Nuclear Research, \\ 141980 Dubna, Russia \\ ${ }^{3}$ Center for Materials Theory, Department of Physics and Astronomy, Rutgers University, \\ Piscataway, NJ 08854, USA \\ E-mail: drchal@fzu.cz
}

Received 11 June 2004, in final form 4 November 2004

Published 10 December 2004

Online at stacks.iop.org/JPhysCM/17/61

\begin{abstract}
We study the two-band degenerate Hubbard model using the fluctuation exchange approximation (FLEX) and compare the results with quantum Monte Carlo (QMC) calculations. Both the self-consistent and the non-self-consistent versions of the FLEX scheme are investigated. We find that, unlike in the oneband case, in the multiband case, good agreement with the quantum Monte Carlo results is obtained within the electron-electron $T$-matrix approximation using the full renormalization of the one-particle propagators. The crossover to strong coupling and the formation of satellites is more clearly visible in the non-self-consistent scheme. Finally we discuss the behaviour of the FLEX for higher orbital degeneracy.
\end{abstract}

(Some figures in this article are in colour only in the electronic version)

\section{Introduction}

Recent progress in the development and application of advanced experimental techniques made available a number of new materials and compounds with strongly correlated electrons that cannot be entirely described within the density functional theory (DFT) $[1,2]$. Some transition metal alloys, cuprates, manganites, heavy-fermion Kondo and mixed-valence systems, as well as lanthanides and transuranium compounds, behave in a way that can neither be explained nor understood within first-principles computational schemes based on local approximations of the correlation-exchange potential in the DFT. It is necessary to take dynamical fluctuations explicitly into account to describe these materials. This can be done best and in a manageable way within the dynamical mean-field theory (DMFT) [3]. The DMFT captures most of the relevant local quantum dynamical effects of electron correlations. Recent progress in the 
development of various impurity solvers for the DMFT has opened a way for combining advanced many-body techniques with $a b$ initio methods to build realistic computational schemes for materials with strongly correlated electrons.

The standard way of combining ab initio calculations with the DMFT is via multiorbital Hubbard models. The input parameters for multiorbital Hubbard models to be solved within the DMFT are determined from ab initio calculations, for example using the linearized muffintin orbital (LMTO) method [4]. No universal scheme for solving the DMFT equations exactly does exist. Hence, one has to resort to approximate impurity solvers to reach quantitative results from the DMFT. We distinguish essentially two kinds of DMFT solvers: numerical schemes and analytical methods based on many-body perturbation theory. The former schemes aim at numerically exact quantitative solutions while the latter aim at analytically controllable schemes. Although analytic methods are not quantitatively as accurate as the numerical solutions, they have an appealing feature in that they offer an analytically controllable approach with direct access to spectral functions on the real frequency axis. Analytic approaches are needed in most situations to complement the numerical solutions so that we can assess the peak structure of spectral functions when performing analytic continuation of numerical results from the imaginary axis of Matsubara frequencies. To gain confidence in approaches based on manybody perturbation theory we should test them in simpler situations and compare their results with available more precise numerical simulations.

In the context of the one-band model, extensive comparisons between perturbative approaches such as the iterated perturbation theory (IPT) and the QMC method have been carried out (see, for example, [5, 3]). It appeared that the non-self-consistent IPT is a rather accurate approximation. Analytic extension of the IPT and second-order perturbation theory via multiple two-particle scatterings, the FLEX, has already been applied to iron and nickel in self-consistent and non-self-consistent forms [6, 7]. However, a critical discussion of the accuracy of this method in multiband situations and comparison with e.g. QMC results have not yet been carried out. It is the aim of this paper to fill this void, and compare diagrammatic schemes with dynamical fluctuations based on two-particle scatterings with finite-temperature quantum Monte Carlo solution of the DMFT. To this end we use a multiorbital Hubbard model with a simplified kinetic energy so that we can focus our attention on the fundamental features of the transition between weak and strong electron couplings in multiorbital Hubbard models.

A typical form of the Hamiltonian used in dynamical extensions of DFT schemes is

$H^{\text {Hubb }}=\sum_{\mathbf{R} \lambda, \mathbf{R}^{\prime} \lambda^{\prime}} t_{\mathbf{R} \lambda, \mathbf{R}^{\prime} \lambda^{\prime}} a_{\mathbf{R} \lambda}^{\dagger} a_{\mathbf{R}^{\prime} \lambda^{\prime}}+\sum_{\mathbf{R}, \lambda, \lambda^{\prime} \lambda^{\prime \prime} \lambda^{\prime \prime \prime}}\left\langle\mathbf{R} \lambda, \mathbf{R} \lambda^{\prime}|V| \mathbf{R} \lambda^{\prime \prime} \mathbf{R} \lambda^{\prime \prime \prime}\right\rangle a_{\mathbf{R} \lambda}^{\dagger} a_{\mathbf{R} \lambda^{\prime}}^{\dagger} a_{\mathbf{R} \lambda^{\prime \prime \prime}} a_{\mathbf{R} \lambda^{\prime \prime}}$,

where $\mathbf{R}$ are lattice site coordinates and $\lambda=(l \sigma)$ are spin-orbital indices. The hopping term $t_{\mathbf{R} \lambda, \mathbf{R}^{\prime} \lambda^{\prime}}$ is determined from ab initio electronic structure calculations and will be replaced in this comparison study with a model dispersion relation diagonal in the spin-orbital indices. The electron interaction is usually considered only between the d electrons, since the effect of the lower orbitals is assumed to be described quite well within the standard DFT. We assume that the local interaction consists only of direct and exchange terms. We approximate the interaction operator with two parameters only: the Hubbard $U$ and the exchange constant $J$. In homogeneous cases (without disorder) we can neglect the lattice coordinate and represent the interaction only with a quadruple of spin-orbital indices:

$$
\left\langle i \sigma j \sigma^{\prime}|V| k \sigma l \sigma^{\prime}\right\rangle \approx \delta_{i k} \delta_{j l}\left(1-\delta_{i j} \delta_{\sigma \sigma^{\prime}}\right) U+\delta_{i l} \delta_{j k}\left(1-\delta_{i j}\right) \delta_{\sigma \sigma^{\prime}} J .
$$

This representation can easily be further simplified to a standard matrix in the spin-orbital indices

$$
v_{\lambda \lambda^{\prime}}=\left(1-\delta_{\lambda \lambda^{\prime}}\right)\left(U-J \delta_{\sigma \sigma^{\prime}}\right)
$$


We use this representation of the electron interaction in our many-body treatment of the multiorbital Hubbard model.

\section{Methods}

\subsection{Many-body dynamical fluctuations}

The effects of the electron interaction on one-particle states are described by the self-energy $\Sigma_{\lambda}$. Dynamical fluctuations are contained in the two-particle vertex function $\Gamma_{\lambda \lambda^{\prime}}$. We denote the four-momenta as $k=\left(\mathbf{k}, \mathrm{i} \omega_{n}\right)$ and $q=\left(\mathbf{q}, \mathrm{i} v_{m}\right)$, and use the Schwinger-Dyson equation to relate the two-particle vertex to the one-particle self-energy. With representation (3) for the electron interaction we can write

$$
\Sigma_{\lambda}(k)=\sum_{\lambda^{\prime}} \frac{1}{\beta N} \sum_{k^{\prime}} v_{\lambda \lambda^{\prime}} G_{\lambda^{\prime}}\left(k^{\prime}\right)\left[1-\frac{1}{\beta N} \sum_{q} G_{\lambda}(k-q) G_{\lambda^{\prime}}\left(k^{\prime}-q\right) \Gamma_{\lambda \lambda^{\prime}}\left(k-q ; q, k^{\prime}-k\right)\right] .
$$

The first term on the rhs of equation (4) is the static Hartree term expressing the self-energy in terms of densities. This term in realistic calculations is normally part of the static local density approximation fixing the static particle densities. We hence suppress the Hartree term and use only the vertex contribution to the self-energy as a generator of dynamical fluctuations missing in the DFT.

The simplest approximation to the vertex function $\Gamma_{\lambda \lambda^{\prime}}$ is the bare interaction $v_{\lambda \lambda^{\prime}}$. Such an approximation corresponds to second-order perturbation theory (SOPT). The vertex is momentum independent. Even in more advanced approximations we will not use the full momentum dependence of the vertex function. In our treatment we resort only to multiple scatterings of two quasiparticles (FLEX) [8]. In this situation the two-particle vertex depends on only a single bosonic four-momentum $q$. This dependence enters the vertex function via a two-particle bubble. When we deal with multiple electron-hole scatterings the bubble is

$$
\Phi_{\lambda \lambda^{\prime}}(q)=\frac{1}{\beta N} \sum_{k} G_{\lambda}(k) G_{\lambda^{\prime}}(k+q) .
$$

The self-energy due to dynamical electron-hole (multiple) scatterings can then be represented as

$$
\Sigma_{\lambda}^{\mathrm{eh}}(k)=\sum_{\lambda^{\prime}} \frac{1}{\beta N} \sum_{q} v_{\lambda \lambda^{\prime}} G_{\lambda^{\prime}}(k+q) \Phi_{\lambda \lambda^{\prime}}(q) \Gamma_{\lambda \lambda^{\prime}}^{\mathrm{eh}}(q) .
$$

In second-order perturbation theory, $\Gamma_{\lambda \lambda^{\prime}}^{\mathrm{eh}}=v_{\lambda \lambda^{\prime}}$. When we sum ladder electron-hole diagrams we obtain for the vertex function the following representation:

$$
\Gamma_{\lambda \lambda^{\prime}}^{\mathrm{eh}}(q)=\frac{v_{\lambda \lambda^{\prime}}}{1+v_{\lambda \lambda^{\prime}} \Phi_{\lambda \lambda^{\prime}}(q)} .
$$

To visualize the above equations we show corresponding diagrams for the vertex $\Gamma^{\mathrm{eh}}$ of the particle-particle type shown in the top row of figure 1 and the corresponding contribution to the self-energy is shown in the bottom row of figure 1 . Note that a small square in all diagrams (figures 1-3) represents the antisymmetrized pair interaction (see [9]).

Analogously we can construct an approximation with multiple electron-electron scatterings where the self-energy can be represented as

$$
\Sigma_{\lambda}^{\mathrm{ee}}(k)=\sum_{\lambda^{\prime}} \frac{1}{\beta N} \sum_{q} v_{\lambda \lambda^{\prime}} G_{\lambda^{\prime}}(q-k) \Psi_{\lambda \lambda^{\prime}}(q) \Gamma_{\lambda \lambda^{\prime}}^{\mathrm{e}}(q) .
$$




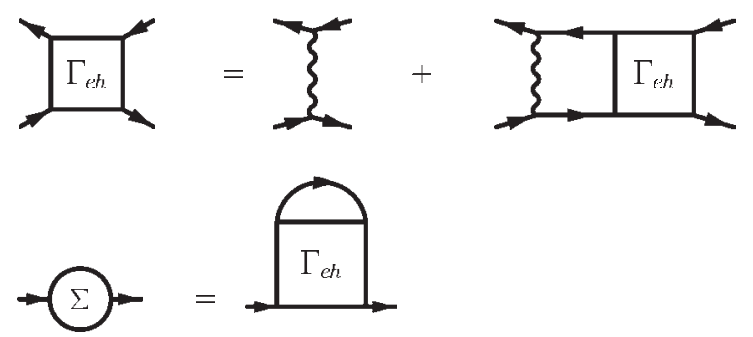

Figure 1. The particle-hole (top row) vertex $\Gamma^{\mathrm{eh}}$ which appears in the FLEX approximation; the definition of the FLEX self-energy constructed with the help of the particle-hole vertex (bottom row)
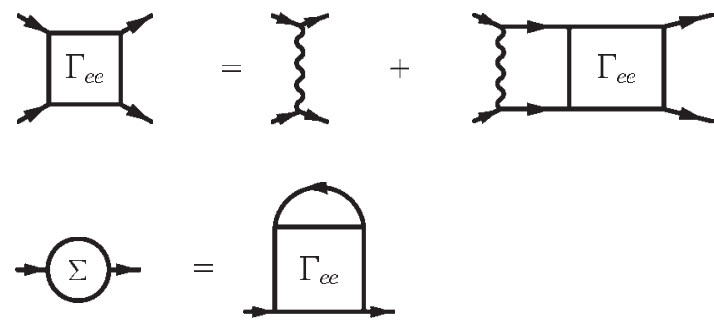

Figure 2. The particle-particle (top row) vertex $\Gamma^{\mathrm{ee}}$ which appears in the FLEX approximation; the definition of the FLEX self-energy constructed with the help of the particle-particle vertex (bottom row).
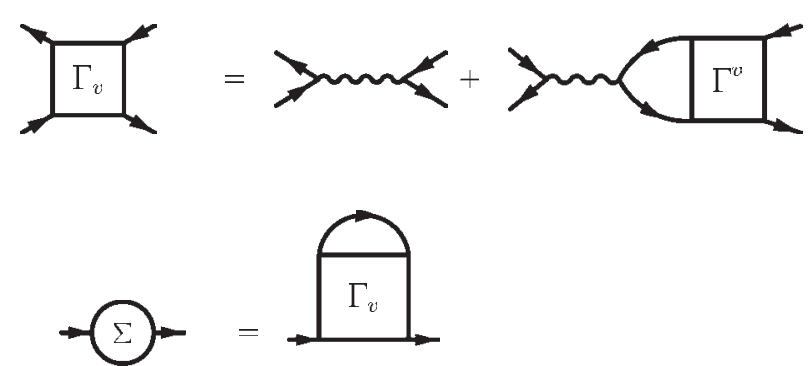

Figure 3. The particle-hole vertex $\Gamma^{\mathrm{v}}$ corresponding to the third scattering channel is given in the top row. The definition of the corresponding self-energy is presented in the bottom row.

Here we have to use a particle-particle bubble

$$
\Psi_{\lambda \lambda^{\prime}}(q)=\frac{1}{\beta N} \sum_{k} G_{\lambda}(k) G_{\lambda^{\prime}}(q-k) .
$$

The two-particle vertex $\Gamma_{\lambda \lambda^{\prime}}^{\mathrm{ee}}$ has the same solution as the electron-hole scattering function, equation (7), where we just replace the bubble $\Phi$ with $\Psi$. The corresponding set of diagrams for the vertex $\Gamma^{\mathrm{ee}}$ and the self-energy is presented in figure 2.

The third channel of two-particle scatterings is the interaction channel where the electron interaction is screened by electron-hole polarization bubbles. The dynamical self-energy due to this renormalization is then represented as

$$
\Sigma_{\lambda}^{\mathrm{v}}(k)=\sum_{\lambda^{\prime}} \frac{1}{\beta N} \sum_{q} v_{\lambda \lambda^{\prime}} G_{\lambda^{\prime}}(k+q) \Phi_{\lambda \lambda^{\prime}}(q) \Gamma_{\lambda \lambda^{\prime}}^{\mathrm{v}}(q),
$$


with

$$
\Gamma_{\lambda \lambda^{\prime}}^{\mathrm{v}}(q)=v_{\lambda \lambda^{\prime}}-\sum_{\lambda^{\prime \prime}} v_{\lambda \lambda^{\prime \prime}} \Phi_{\lambda^{\prime \prime} \lambda^{\prime \prime}}(q) \Gamma_{\lambda^{\prime \prime} \lambda^{\prime}}^{\mathrm{v}}(q)
$$

Corresponding diagrams are presented in figure 3 . We can treat each channel independently or add all three channels to assess the effect of dynamical fluctuations on the electron self-energy. In the latter case, however, we have to subtract twice the contribution from the second order, since it is identical in all three channels.

The idea of the DMFT is to neglect the momentum dependence of the one-electron propagators in the contributions to the self-energy. We hence use only the local parts of the one-electron propagators, i.e., $G_{\lambda}\left(\mathbf{k}, \mathrm{i} \omega_{n}\right) \rightarrow N^{-1} \sum_{\mathbf{k}} G_{\lambda}\left(\mathbf{k}, \mathrm{i} \omega_{n}\right)=G_{\lambda}\left(\mathrm{i} \omega_{n}\right)$. Then, all the above formulae hold with the replacements $k \rightarrow \mathrm{i} \omega_{n}, q \rightarrow \mathrm{i} v_{m}$ for fermionic and bosonic momenta, respectively.

The advantage of analytic approaches with multiple two-particle scatterings is the knowledge of the explicit analytic structure of the self-energy. Hence all the above results can be explicitly analytically continued to real frequencies using a straightforward procedure. We explicitly mention only the result for the two-particle electron-hole bubble:

$\Phi_{\lambda \lambda^{\prime}}(z)=-\int_{-\infty}^{\infty} \frac{\mathrm{d} \omega}{\pi} f(\omega-\mu)\left[G_{\lambda^{\prime}}(\omega+z) \operatorname{Im} G_{\lambda}\left(\omega_{+}\right)+G_{\lambda}(\omega-z) \operatorname{Im} G_{\lambda^{\prime}}\left(\omega_{+}\right)\right]$.

Up to now we have used the fully renormalized one-electron propagators in the perturbation theory as demanded by conservation laws. However, when we are interested in the one-electron properties of the system, we can relax the demands of thermodynamic consistency and replace the fully renormalized propagator with a partially renormalized one:

$$
G^{-1}(z) \longrightarrow \mathcal{G}_{0}^{-1}(z)=G^{-1}(z)+\Sigma(z)+\tilde{\mu},
$$

where in the expression for $\mathcal{G}_{0}$ we used an additional shift of the impurity level to satisfy Luttinger's theorem following [10]. This propagator goes over into the bare propagator in the atomic limit and does not contain long energy tails due to frequency convolutions in the definition of the self-energy.

This partially non-self-consistent scheme resembles IPT. Hence a better description of the transition from weak to strong coupling regimes at the one-particle level, including the metalinsulator transition (MIT), is expected from the IPT than from the conserving scheme with fully renormalized propagators, where the metal-insulator transition at half-filling is known to be missing.

\subsection{The quantum Monte Carlo case}

Among many methods used to solve the impurity problem we choose the quantum Monte Carlo method [11] to benchmark FLEX as a potential candidate impurity solver. There are well known advantages and disadvantages of the QMC method and our choice is spurred by the fact that despite being slower than other methods, the QMC approach is a well controlled, numerically exact method. As an input the QMC procedure has the Weiss function $\mathcal{G}_{0}(\tau)$ and as an output it produces the Green function $G(\tau)$. We remind the reader of the major steps taken in the QMC procedure. Usually one starts with an impurity effective action $S$ :

$S_{\text {eff }}=-\int_{0}^{\beta} \mathrm{d} \tau \mathrm{d} \tau^{\prime} \sum_{\alpha} c_{\alpha}^{\dagger}(\tau) \mathcal{G}_{0_{\alpha}}^{-1}\left(\tau, \tau^{\prime}\right) c_{\alpha}\left(\tau^{\prime}\right)+\frac{1}{2} \int_{0}^{\beta} \mathrm{d} \tau \sum_{\alpha, \alpha^{\prime}} U_{\alpha \alpha^{\prime}} n_{\alpha}(\tau) n_{\alpha^{\prime}}(\tau)$,

where $\left\{c, c^{\dagger}\right\}$ are fermionic annihilation and creation operators of the lattice problem, $\alpha=\{m, \sigma\}$. 
The first thing we should do with the action (14) is to discretize it in imaginary time with time step $\Delta \tau$ so that $\beta=L \Delta \tau$, and $L$ is the number of time intervals:

$$
S_{\text {eff }} \rightarrow \sum_{\alpha, \tau \tau^{\prime}} c_{\alpha}^{\dagger}(\tau) \mathcal{G}_{0}^{-1}\left(\tau, \tau^{\prime}\right) c_{\alpha}\left(\tau^{\prime}\right)+\frac{1}{2} \sum_{\alpha, \alpha^{\prime}} U_{\alpha \alpha^{\prime}} n_{\alpha}(\tau) n_{\alpha^{\prime}}(\tau)
$$

The next step is to get rid of the interaction term $U$ by replacing it by summation over Ising-like auxiliary fields. The decoupling procedure is called the Hubbard-Stratonovich transformation $[12,13]$ :

$$
\exp \left\{-\Delta \tau\left\{U_{\alpha \alpha^{\prime}} n_{\alpha} n_{\alpha^{\prime}}-\frac{1}{2}\left(n_{\alpha}+n_{\alpha^{\prime}}\right)\right\}\right\}=\frac{1}{2} \sum_{S_{\alpha \alpha^{\prime}}= \pm 1} \exp \left\{\lambda_{\alpha \alpha^{\prime}} S_{\alpha \alpha^{\prime}}\left(n_{\alpha}-n_{\alpha^{\prime}}\right)\right\}
$$

where $\cosh \lambda_{\alpha \alpha^{\prime}}=\exp \left(\frac{\Delta \tau U_{\alpha \alpha^{\prime}}}{2}\right)$ and $S_{\alpha \alpha^{\prime}}\left(\tau_{l}\right)$ are auxiliary Ising fields at each time slice.

In the one-band Anderson impurity model we have only one auxiliary Ising field $S\left(\tau_{l}\right)= \pm 1$ at each time slice, whereas in the multiorbital case the number of auxiliary fields is equal to the number of $\alpha, \alpha^{\prime}$ pairs. Applying the Hubbard-Stratonovich transformation at each time slice we bring the action into a quadratic form with the partition function

$$
Z=\operatorname{Tr}_{\left\{S_{\alpha \alpha^{\prime}}(\tau)\right\}} \prod_{\alpha} \operatorname{det} G_{\alpha,\left\{S_{\alpha \alpha^{\prime}}(\tau)\right\}}^{-1}
$$

where the Green function in terms of auxiliary fields $G_{\alpha}^{-1}$ reads

$$
G_{\alpha,\left\{S_{\alpha \alpha^{\prime}}\right\}}^{-1}\left(\tau, \tau^{\prime}\right)=\mathcal{G}_{0_{\alpha}}^{-1}\left(\tau, \tau^{\prime}\right) \mathrm{e}^{V}-\left(\mathrm{e}^{V}-1\right) \delta_{\tau, \tau^{\prime}},
$$

with the interaction matrix

$$
V_{\tau}^{\alpha}=\sum_{\alpha^{\prime}(\neq \alpha)} \lambda_{\alpha \alpha^{\prime}} S_{\alpha \alpha^{\prime}}(\tau) \sigma_{\alpha \alpha^{\prime}}
$$

where

$$
\sigma_{\alpha \alpha^{\prime}}= \begin{cases}+1 & \text { for } \alpha<\alpha^{\prime} \\ -1 & \text { for } \alpha>\alpha^{\prime}\end{cases}
$$

Once the quadratic form is obtained, one can apply Wick's theorem at each time slice and perform the Gaussian integration in Grassmann variables to get the full interacting Green function:

$$
G_{\alpha}\left(\tau, \tau^{\prime}\right)=\frac{1}{Z} \operatorname{Tr}_{\left\{S_{\alpha \alpha^{\prime}}\right\}} G_{\alpha,\left\{S_{\alpha \alpha^{\prime}}\right\}}\left(\tau, \tau^{\prime}\right) \prod_{\alpha^{\prime}} \operatorname{det} G_{\alpha^{\prime},\left\{S_{\alpha \alpha^{\prime}}\right\}}^{-1}
$$

To evaluate the summation in equation (20) one uses Monte Carlo stochastic sampling. The product of determinants is interpreted as the stochastic weight and auxiliary spin configurations are generated by a Markov process with probability proportional to their statistical weight. A more rigorous derivation can be found elsewhere [3, 13].

Since the QMC method produces results on the imaginary time axis $\left(G\left(\tau_{m}\right)\right.$ with $\left.\tau_{m}=m \Delta \tau, m=1 \ldots L\right)$ and the DMFT self-consistency equations make use of the frequency dependent Green functions and self-energies, we must have an accurate method to compute Fourier transformations from the time to the frequency domain. This is done by representing the functions in the time domain by cubic splined functions which should go through the original points with the condition of continuous second derivatives imposed. Once we know the cubic spline coefficients we can compute the Fourier transformation of the splined functions analytically. 


\section{Results and discussion}

To compare analytic solutions with numerical ones, we employed a simple model with two degenerate bands, i.e., four spin orbitals per site, and assumed a semi-elliptic density of states (DOS), $\rho_{0}(E)=\frac{2}{\pi} \sqrt{1-E^{2}}$. For simplicity we resorted to the case $J=0$ and non-magnetic solutions. We tested second-order perturbation theory together with multiple scatterings from the electron-hole and electron-electron interaction channels. Both types of self-consistency were considered, i.e., the full conserving and the partial one with the bath function $\mathcal{G}_{0}$, defined in the IPT equation (13). All approximations were analytically continued to real frequencies before being evaluated numerically. The maximum entropy method was used for analytical continuation to the real axis. We tested the maximum entropy method against other highfrequency, more reliable methods such as the non-crossing approximation and the one-crossing approximation and found satisfactory agreement, understandable within the limitations of all methods used. A simple iteration procedure with a suitably chosen mixing of the old and new self-energy led to well converged results for moderate values of the pair interaction $U$. The details of the numerical implementation of multiorbital FLEX-type approximations were described elsewhere [6, 7, 14-16].

First, we compared the analytical approximations among themselves for an intermediate value of the interaction strength, $U=2$. All energies are given in units of the half-bandwidth of the non-interacting DOS, $D=1$. We also wrote two different FLEX programs on real and imaginary axes and used 9000 and 4096 points on the corresponding frequency domain with large enough frequency cut-off $(10 D)$ for temperature $T=1 / 16$. Using the conserving full self-consistency we found that results from SOPT and the electron-electron $T$-matrix approximation, TMA, are quite close to each other. The addition of the electron-hole TMA channel changes the result significantly. The quasiparticle width becomes substantially reduced, as shown in figure 4 . This strong band narrowing is unphysical. We did not include the third channel, screening by the electron-hole polarization bubbles, explicitly in this figure, since it contributes quantitatively and qualitatively similarly to the electron-hole ladder. The reason that the electron-hole scatterings do not improve at second order is quite clear. At the mean-field level an instability towards the local moment state with non-zero $\left\langle n_{\uparrow}\right\rangle-\left\langle n_{\downarrow}\right\rangle$ appears at some critical interaction. This instability is totally artificial because the conduction electrons screen the local moment (Kondo effect). In the non-self-consistent FLEX approach including both electron-electron and electron-hole TMA, this instability also occurs when the denominator in the above equation contains a pole at zero frequency, for example for the two-band Hubbard model with the half-bandwidth equal to the one the instability happens at, $U=0.7$, for temperature $T=1 / 16$. So the presence of this artificial instability will push the results obtained by the non-self-consistent FLEX approach including electron-hole TMA away from the right answer. For the self-consistent FLEX approach including electron-hole TMA, the instability happens for $U$ slightly larger than two for the same model, indicating a tendency towards a phase transition from the paramagnetic to a ferromagnetic solution in the lattice case which is unphysical for the impurity problem. Thus, this unphysical divergence overestimates the contribution of the electron-hole scatterings and the result worsens with increasing interaction strength. To improve on this, one can use Kanamori's [17] observation that the particle-hole bubbles should interact not with the bare interaction but with an effective one screened by the particle-particle ladder,i.e. replace $U$ by $U_{\text {eff }}$ equal to the electron-electron $T$-matrix taken at zero frequency.

As is known from the single-band situation, neither of the FLEX-type approximations is able to trace the emergence of satellite Hubbard bands and the metal-insulator transition. The high-energy spectrum is shapeless and broadened due to energy convolutions. This situation 


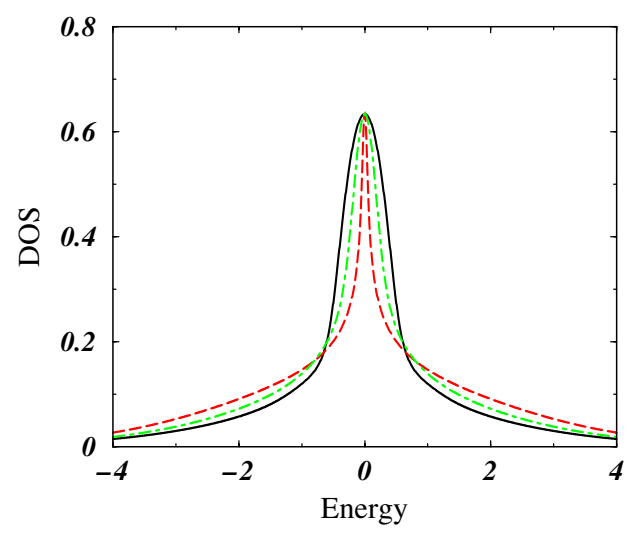

Figure 4. Densities of states for $U=2, n=2$ (half-filled case) calculated within the second-order perturbation theory (dot-dashed curve), electron-electron TMA (solid curve), and electron-hole TMA (dashed curve). The oneparticle propagators are fully renormalized in the selfconsistent calculations $(\Sigma=\Sigma[G])$.

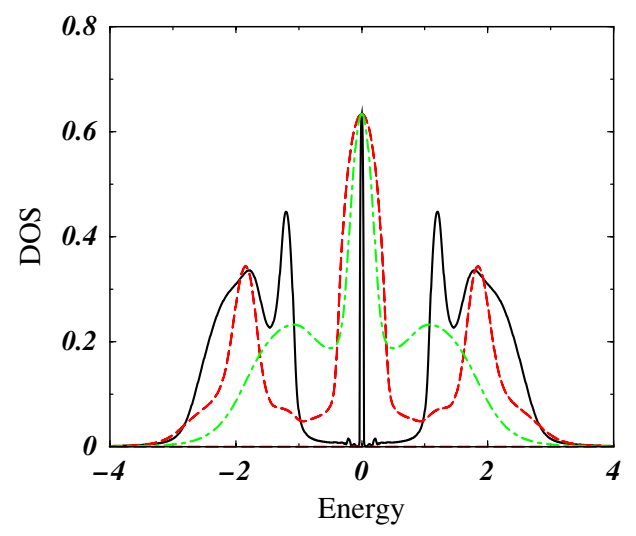

Figure 5. Densities of states for $U=2, n=2$ (half-filled case) calculated within the second-order perturbation theory (solid curve), the electron-electron TMA (dashed curve), and the electron-hole TMA (dot-dashed curve). The one-particle propagators are partially renormalized by using the iterated perturbation method $\left(\Sigma=\Sigma\left[\mathcal{G}_{0}\right]\right)$.

changes dramatically if we keep only the topological self-consistency of the one-particle propagators obtained from the IPT; see figure 5. We can see that second order and the electronelectron TMA produces the Hubbard satellite bands in their correct positions. The SOPT scatterings add an additional internal structure to the satellite bands. While the satellite bands gain more weight than they actually have at this interaction strength, the central quasiparticle peak weight is strongly reduced in non-self-consistent or partly self-consistent approximations. Too much quasiparticle weight is transferred to the Hubbard bands.

In the electron-hole scattering channel we get slight changes in the positions of the Hubbard bands, and a slight decrease of the quasiparticle width. Even for rather weak interactions $U<1$ one observes nearly total disappearance of the quasiparticle peak when including the electron-hole scattering channel. Due to this unphysical behaviour at moderate electron coupling and the instability towards the local state formation, we leave the electronhole scattering channel out of further considerations. There is no way at the FLEX level to improve upon the results from the electron-electron scattering channel. The best we could do was to add contributions from all three distinct channels. But we have to subtract the secondorder contribution to the self-energy which dominates at moderate and intermediate couplings so that we obtain a negative density of states at the Fermi energy. The only real improvements can be reached via a new self-consistent coupling of the electron-electron and electron-hole channels of the parquet type. Since we are not yet able to implement parquet-type selfconsistency in multiorbital models and have to stay within FLEX, we compare only results of analytical approximations based on second-order perturbation theory and the electron-electron $T$-matrix approach against QMC data.

To compare the analytic FLEX results with quantum Monte Carlo simulations, we use both versions of the self-consistency. In figure 6 the density of states at $U=1$ calculated within the electron-electron $T$-matrix approximation is shown along with the one deduced from the QMC approach. As one can see from the plot, the conserving TMA fits better to the quasiparticle peak than the TMA with the IPT bath Green function $\mathcal{G}_{0}$. In addition, the IPT TMA seems to overemphasize the role of the satellite peaks at weak coupling, as there are no satellites in the QMC solution for this coupling strength. If we increase the interaction 


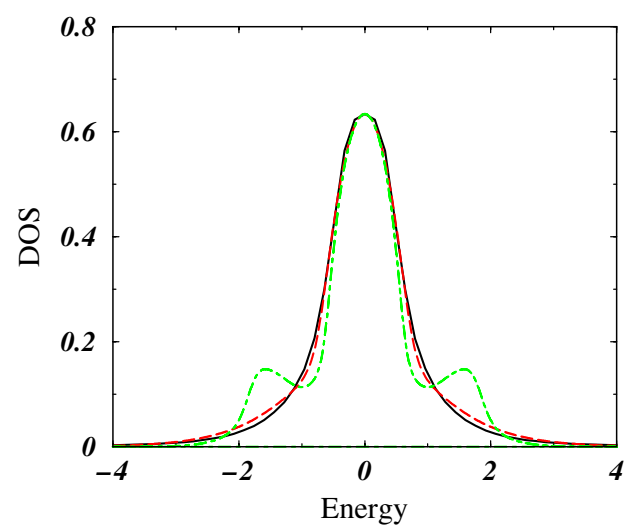

Figure 6. Densities of states for $U=1.0, n=2$ (half-filled case) calculated within the electron-electron TMA using the full renormalization of the one-particle propagators (dashed curve) and partial renormalization using the IPT (dot-dashed curve) compared with the result from the quantum Monte Carlo method (solid curve).

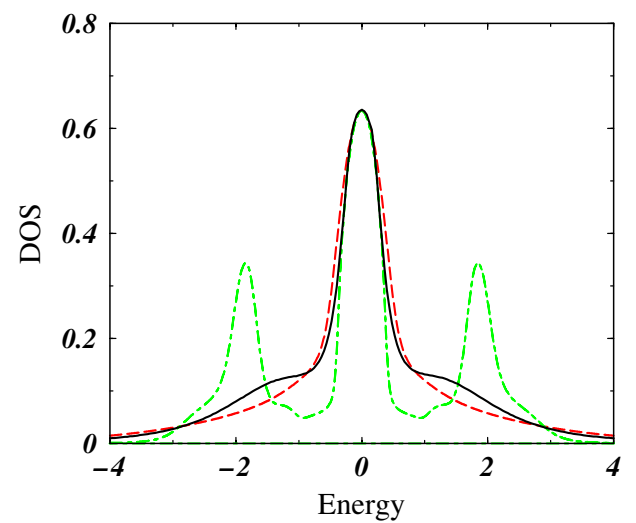

Figure 7. Densities of states for $U=2.0, n=2$ (half-filled case) calculated within the electron-electron TMA using the full renormalization of the one-particle propagators (dashed curve) and partial renormalization using the IPT (dot-dashed curve) compared with the result from the quantum Monte Carlo method (solid curve).

to $U=2$ (see figure 7) and compare the same curves as in the previous plot, we notice that the conserving TMA still reproduces the quasiparticle peak very well, whereas the IPT TMA retains the tendency of reducing the central peak in favour of the satellites. At this coupling the satellites are formed also in the QMC solution but not as strongly as the IPT TMA predicts. Notice that the positions of the satellites reproduced by the IPT solution are rather close to the ones coming from the QMC approach.

As a summary of the FLEX for the symmetric case of the multiband Hubbard model we present the dependence of the quasiparticle residue, $Z$, on the interaction strength, $U$. It is clear that the closer the $Z(U)$ curve for a particular approximation to the QMC data, the better the approximation works in reproducing the quasiparticle properties of the system, including the central peak weight and width. In figure 8 we present the following curves: QMC data are plotted as a solid curve with open circle symbols; the results from the second-order perturbation theory in the cases of fully renormalized one-particle propagators in the self-consistent calculations $(\Sigma=\Sigma[G])$ and ones partially renormalized by using the iterated perturbation method $\left(\Sigma=\Sigma\left[\mathcal{G}_{0}\right]\right)$ are given by dashed and dot-dashed curves respectively. Concentrating on small and intermediate values of the interaction, we can see that in the first case of the full renormalization of the propagators the situation is significantly better, as the dashed curve is much closer to the QMC one than the dot-dashed curve. The electron-electron TMA gives even better results than those obtained from SOPT. Both the electron-electron TMA curves, for the case of the full self-consistency $(\Sigma=\Sigma[G])$, plotted as a solid curve, and the partial one $\left(\Sigma=\Sigma\left[\mathcal{G}_{0}\right]\right)$, plotted as a dotted curve, lie much closer to the QMC data than the SOPT curves (for interactions smaller than or equal to $U=2$ ). From the two electron-electron TMA curves we make a decision in favour of the one obtained using the fully renormalized one-particle propagators in the self-consistency procedure. This result differs from the findings in the case of the one-band Hubbard model, where partially renormalized propagators gave better agreement with Monte Carlo results. Note that the FLEX-type approximations follow the Monte Carlo quasiparticle weight only for weak and moderate interaction strengths, $U \leqslant 2$. All approximations except for the IPT go wrong near and beyond the expected metal-insulator transition. 


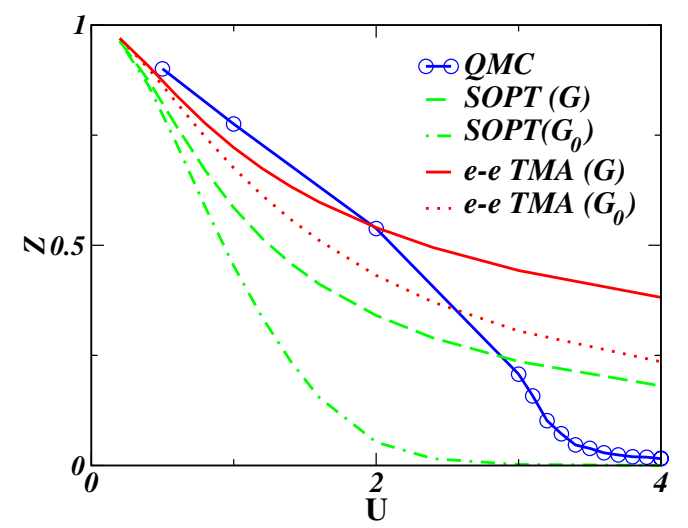

Figure 8. The dependence of the quasiparticle residue $Z$ on the Coulomb repulsion $U$ calculated within the electron-electron TMA (solid and dotted curves) and the second-order perturbation theory (dashed and dot-dashed curves) using the full renormalization of the one-particle propagators and partial renormalization using the IPT respectively. QMC results are plotted as a solid curve with circle symbols.

In figure 9 we study the dependence of the DOS, calculated by different methods, on the orbital degeneracy. We calculated the DOS within two- and three-band Hubbard models in the half-filled case using the QMC approach as the tester against the two best FLEX approximations which we found, namely, the electron-electron TMA with full self-consistency $(\Sigma=\Sigma[G])$ and the partial one $\left(\Sigma=\Sigma\left[\mathcal{G}_{0}\right]\right)$. One can almost immediately notice the main difference between the QMC and FLEX results. The width of the quasiparticle (QP) peak in the QMC calculation slightly increases with the degeneracy. This is to be expected as, while the degeneracy grows, the critical $U$ also increases, which takes the curve for the $Z$ versus $U$ dependence (see figure 8) higher for larger degeneracy at any particular repulsion. As an example, the quasiparticle residue against repulsion at $U=2$ for degeneracy $N=6$ will be above the curve in figure 8 corresponding to degeneracy $N=4$. FLEX results show the opposite dependence: the QP width decreases with increasing degeneracy. We should also note that this wrong tendency is stronger for the partial (IPT) self-consistency. The reason for such behaviour can lie in the limited set of diagrams treated in FLEX, resulting in insufficient screening for higher degeneracy.

The self-consistent FLEX scheme was found to reproduce quite well the central quasiparticle peak for two and three bands at weak and medium interaction strengths. It is, however, important to stress that this cannot persist to very large degeneracy. It has been shown [18] that in the exact solution of the DMFT equations for large $N$ the critical $U$ at which the Mott transition takes place at zero temperature scales linearly with $N$. This implies that for fixed $U$ the quasiparticle residue, which has an approximate expression $Z=1-U / U_{\mathrm{c} 2}$, increases and eventually approaches unity with increasing orbital degeneracy. This remarkable screening effect in the multiorbital degenerate Hubbard model is not captured by the FLEX approach, which displays the opposite trend, as can be seen in figure 10 .

When we move off the electron-hole symmetric case, the situation changes. The quasiparticle peak is no longer as strongly suppressed in the electron-hole scattering channels. Moreover, the conserving TMA starts to develop a satellite peak. Figure 11 demonstrates this trend for $n=0.8$. We perceive almost no difference in the form of the central peak between the two different self-consistent versions of the multiple electron-electron scatterings. The IPT version retains its tendency to overemphasize the width of the satellite peaks and produces 


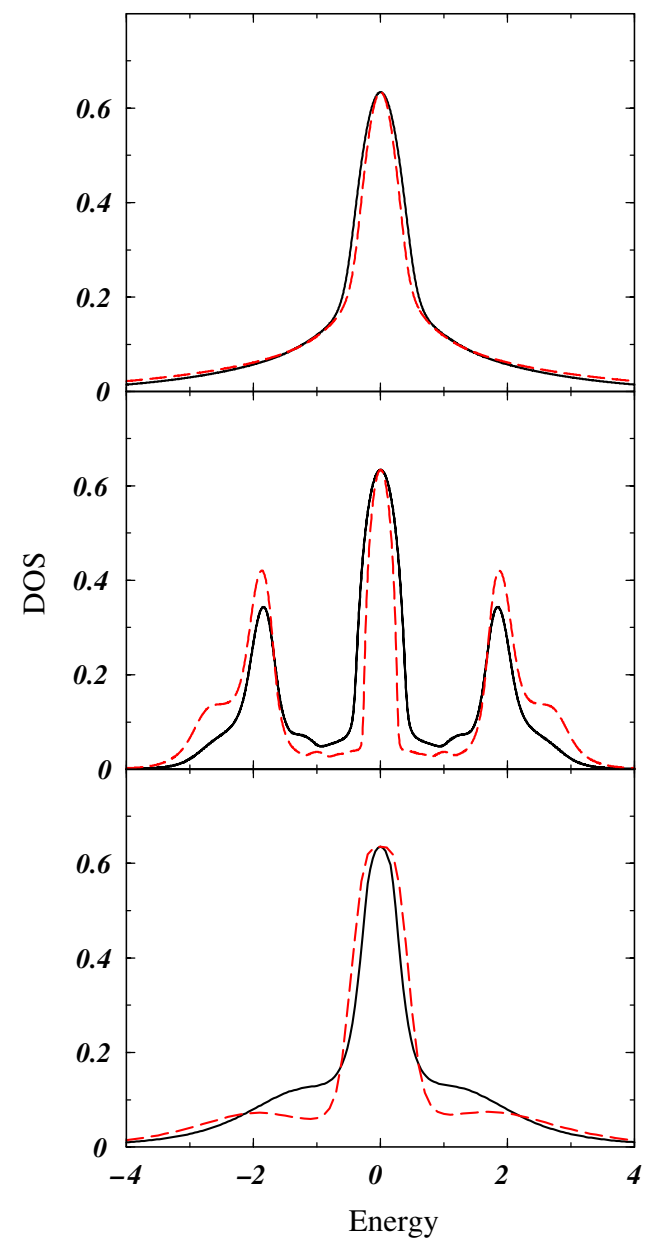

Figure 9. Densities of states for $U=2.0, n=2$ in the two-band model (solid curves) and $n=3$ in the three-band model (dashed curves) — both cases corresponding to the half-filled case-calculated within the electron-electron TMA using the full renormalization of the one-particle propagators (upper panel) and partial renormalization using the IPT (middle panel) compared with the result from the quantum Monte Carlo method (lower panel).

an incorrect position of the upper Hubbard band. The conserving TMA shows a shoulder behaviour where the QMC approach displays the hole satellite. The less pronounced electron peak cannot be traced either in the IPT TMA or in the conserving TMA.

To explore a wider range of parameters away from half-filling, we plot the quasiparticle residue dependence on the filling in figure 12. With the incrementation of filling $n$, the scattering rate is increased as the number of particles which can scatter a particular electron in the system grows. Therefore it is natural to expect reduction of the quasiparticle residue as a function of $n$ at least for filling $n<1$. As one can see from the plot, the relative positions of the curves are not changed in comparison with figure 8 and one reaches the conclusion that the electron-electron TMA fits best to the QMC data. In addition, one can conclude that for densities $n<1$ both methods (the self-consistent and the non-self-consistent electron-electron TMA) work very well for describing QMC data, while for $n>1$ we observe a deviation of FLEX from QMC data indicating the necessity of taking into account other scattering channels. 


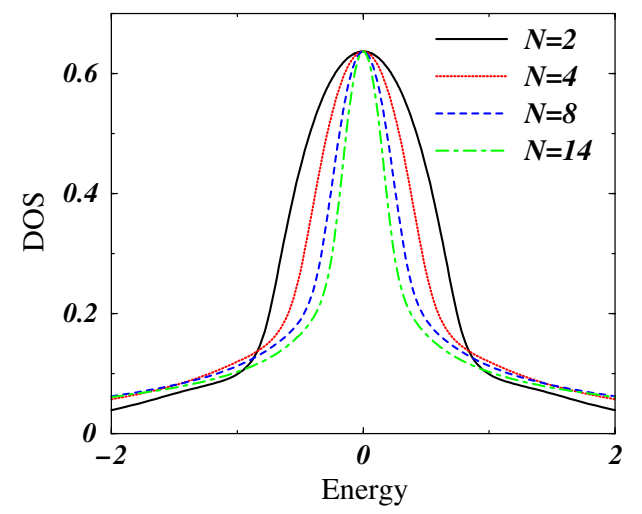

Figure 10. Densities of states for $U=2.0$ ( $n$ is kept at half-filling) calculated within the electron-electron TMA using the full renormalization of the one-particle propagators for different degeneracies (see the legend).

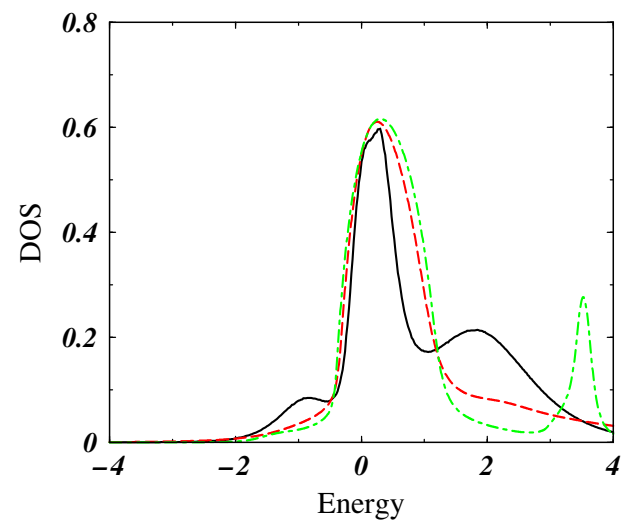

Figure 11. Densities of states for $U=2.0, n=0.8$ (partially filled band) calculated within the electron-electron TMA using the full renormalization of the one-particle propagators (dashed curve) and partial renormalization using the IPT (dot-dashed curve) compared with the result from the quantum Monte Carlo method (solid curve).

The minima in $Z(n)$ at $n=1$ and 2 observed in QMC data become even more pronounced with increasing $U$ and indicate the trace of the metal-insulator transition which appears for the two-band Hubbard model at $U \approx 3.5$, as one can see from figure 8 . As we mentioned above, the FLEX fails to reproduce the MIT, which is also reflected in an almost monotonic decrease of the quasiparticle weight for all densities including the interval between $n=1$ and 2 .

We mention in passing that the one-band situation is exceptional because the exact evaluation of the one-particle Green function shows that the interaction is not efficiently screened. Therefore, the non-self-consistent version of SOPT works well. Furthermore, in the one-band case, the SOPT form combined with the DMFT self-consistency condition produces the correct atomic limit [3]; this is not the case in the degenerate situation. In the multiorbital case, the effective interaction becomes more screened with increasing degeneracy. The selfconsistent version of FLEX attempts to capture this effect, and in fact it does it slightly better than the self-consistent SOPT. The non-self-consistent version of SOPT is less good because it does not screen the interaction at all. 


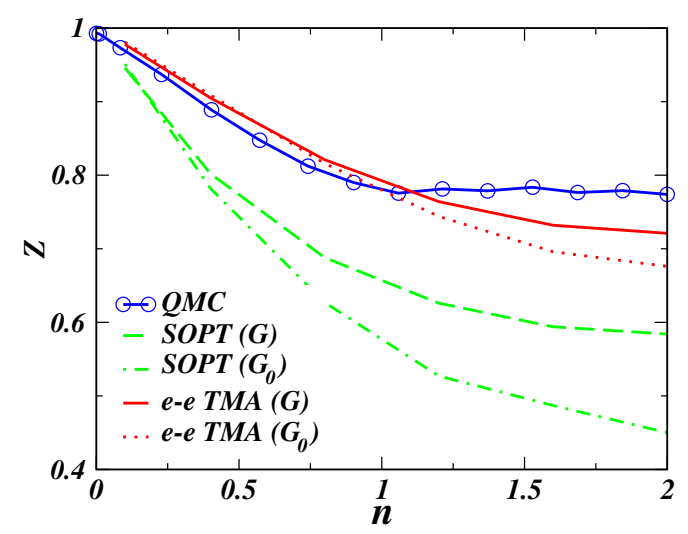

Figure 12. The dependence of the quasiparticle residue $Z$ on the filling $n$ for $U=1$ calculated within the electron-electron TMA (solid and dotted curves) and the second-order perturbation theory (dashed and dot-dashed curves) using the full renormalization of the one-particle propagators and partial renormalization using the IPT respectively. QMC results are plotted as a solid curve with circle symbols.

\section{Conclusion}

In summary, we compared a multiband implementation of the FLEX procedure within the framework of the two-band model with a semi-elliptic non-interacting DOS against the quantum Monte Carlo method. The results obtained indicate that the best agreement with the QMC data is reached for the electron-electron TMA and with fully renormalized oneparticle propagators, $\Sigma=\Sigma[G]$. This conclusion is restricted to small energies within the quasiparticle peak and to the region of moderate Coulomb interaction $(U \leqslant 2)$. Our finding is somewhat different from that for the one-band Hubbard model, where the best match for the same interaction strengths was found for the one-particle propagators partially renormalized by using the iterated perturbation method $\left(\Sigma=\Sigma\left[\mathcal{G}_{0}\right]\right)$. In the multiband situation for band degeneracy that is not high and small to intermediate $U$, the self-consistent version describes the quasiparticle features in good agreement with the QMC approach. On the other hand, there is no hint in the self-consistent calculation of an incipient Mott transition, as in the one-band case [19], while the non-self-consistent scheme clearly gives a hint that a Mott transition will take place, albeit at a very small unphysical value of $U$, due to an inappropriate screening of this interaction.

Insufficient screening due to the limited set of diagrams treated in FLEX results in a quasiparticle weight underestimation with increasing degeneracy in the system. For the nonsymmetric case, FLEX has a tendency to overestimate the QP width and underestimate the Hubbard band weight, given their correct position in the case of the conserving TMA. Our results, found for an impurity solver used within the DMFT, have a wider range of validity as they are naturally applicable to the multiorbital Anderson model as well.

\section{Acknowledgments}

The research was carried out by VD, VJ and JK within the project AVOZ1-010-914 of the Academy of Sciences of the Czech Republic and supported in part by grant A1010203 from the Grant Agency of the Academy of Sciences of the Czech Republic. We (VO, XD, KH and 
GK) acknowledge support from grant NSF-INT9907893, US-Czech Materials Research on Many-Body Correlations in Calculations of Realistic Electronic Structure of Solids. We would like to thank A Lichtenstein for extensive and fruitful discussions of the workings of FLEX. We also would like acknowledge warm hospitality extended to four of us (VJ, VO, XD and GK) during our stay at Kavli Institute for Theoretical Physics during the workshop 'Realistic Theories of Correlated Electron Materials' where part of this work was carried out. The work was supported by the NSF grant DMR-0096462.

\section{References}

[1] Hohenberg P and Kohn W 1964 Phys. Rev. 136 B864

[2] Kohn W 1999 Rev. Mod. Phys. 711253

[3] Georges A, Kotliar G, Krauth W and Rozenberg M 1996 Rev. Mod. Phys. 6813

[4] Turek I, Drchal V, Kudrnovský J, Šob M and Weinberger P 1997 Electronic Structure of Disordered Alloys, Surfaces and Interfaces (Boston-London-Dordrecht: Kluwer-Academic/Plenum)

[5] Kajueter H and Kotliar G 1996 Phys. Rev. Lett. 77 131-4

[6] Drchal V, Janiš V and Kudrnovský J 1999 Electron Correlations and Materials Properties ed A Gonis et al (New York: Kluwer-Academic/Plenum) p 273

[7] Drchal V, Janiš V and Kudrnovský J 1999 Phys. Rev. B 6015664

[8] Bickers N E and Scalapino D J 1989 Ann. Phys. 193206

[9] Abrikosov A A, Gorkov L P and Dzyaloshinski I E 1975 Methods of Quantum Field Theory in Statistical Physics (New York: Dover)

[10] Kajueter H 1996 PhD Thesis Rutgers University Graduate School, New Brunswick, NJ

[11] Hirsch J E and Fye R M 1986 Phys. Rev. Lett. 562521

[12] Hirsch J E 1983 Phys. Rev. B 284059

[13] Takegahara K 1992 J. Phys. Soc. Japan 621736

[14] Drchal V, Janiš V and Kudrnovský J 2003 Electron Correlations and Materials Properties vol 2, ed A Gonis et al (New York: Kluwer Academic/Plenum) p 341

[15] Katsnelson M I and Lichtenstein A I 1999 J. Phys.: Condens. Matter 111037

[16] Katsnelson M I and Lichtenstein A I 2000 Phys. Rev. B 618906

[17] Kanamori J 1963 Prog. Theor. Phys. 30275

[18] Florens S, Georges A, Kotliar G and Parcollet O 2002 Phys. Rev. B 66205102

[19] Menge B and Müller-Hartmann E 1991 Z. Phys. B 82237 